\title{
Development of smart devices to monitor goods using wireless sensor network and internet of things
}

\section{André F. S. Massa*, Leandro T. Manera, Maurício M. Donatti, Orlando F. Lima Jr., Ana P. Noletto}

\begin{abstract}
This work intends to develop electronic devices to solve real problems of monitoring transportable goods. The lowpower mesh network end-devices containing sensors are used to measure environmental and physical properties, as temperature and humidity. Afterwards, radios send all the information to a coordinator module through Zigbee protocol and then to a data center using Wi-Fi. Finally, those data can be used by logistic companies to make decisions and improve their supply chain management.
\end{abstract}

\section{Key words:}

Electronics, Internet of Things (IoT), Wireless Sensor Network (WSN).

\section{Introduction}

The continuous improvement of wireless sensor network made it possible its utilization in many different services, as in monitoring the temperature in critical places ${ }^{1}$.

Nowadays, several logistic enterprises monitor their goods in order to provide better services. Nevertheless, there is still too much waste of sensitive goods around the world due to harsh environmental conditions of transportation and storage. In Brazil, $50 \%$ of the food is wasted during transportation and handling ${ }^{2}$.

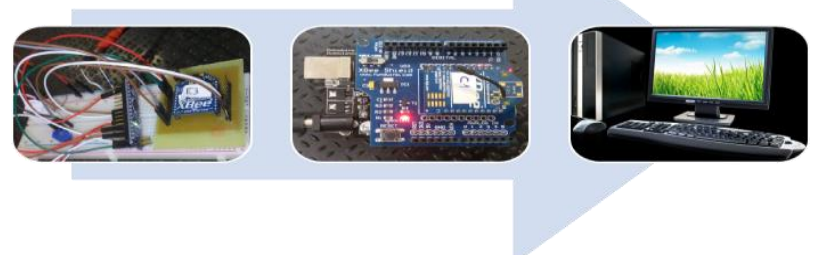

Image 1. Structure of the project; End-device (left), coordinator (middle), data center (right).

The objective of this project is to develop a low-power device to read humidity and temperature, concentrate those information via wireless communication in a coordinator device and then transfer those data to a supply chain company data center, as shown in Image 1.

The low power end-device wakes-up, acquires the temperature and humidity, sends a command to wake-up the radio and transmit the information to the coordinator. Then, the coordinator sends the data to a database via Wi-Fi.

\section{Results and Discussion}

Firstly, we developed a temperature reading device using DS18B20 sensors, Arduino Uno and Ethernet Shield to send data from a refrigerator to a database located at ITAL (Institute of Food Technology) in Campinas, as shown in Image 2.

Afterwards, we developed the wireless low-power enddevice, using the low-cost digital temperature-humidity sensor DHT22, the platform Arduino Nano (based on the Atmel ATMEGA328p) and the XBee Pro Serie 2 radio.

Actually, the low-power end-device consumes a mean current of $53 \mathrm{~mA}$ in sleep mode and works for 5 days using a li-ion battery with no need to recharge.

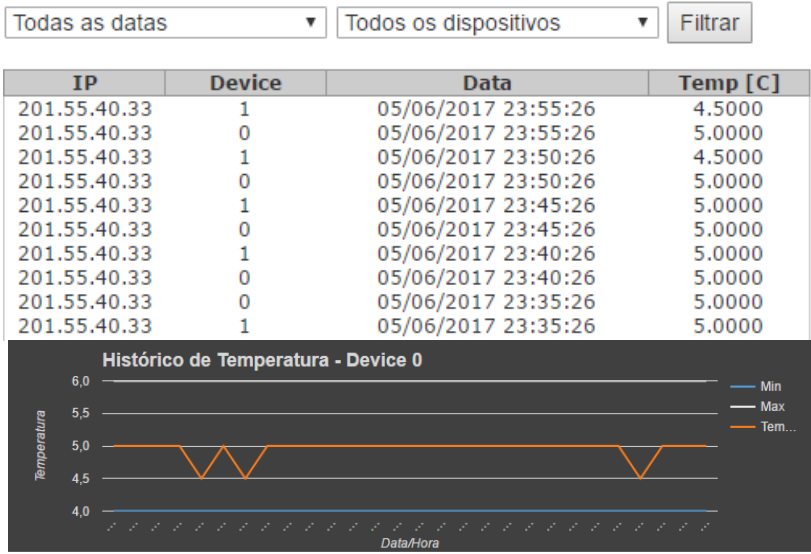

Image 2. ITAL database history.

\section{Conclusions}

The current system works properly and drives all sensors information to the datacenter. Nevertheless, the enddevice power consumption can still be improved, replacing the microcontroller to Arduino Pro Mini and making some adaptations. It is possible to reach a current consumption of less than $10 \mathrm{~mA}$ and is able to work for 25 days with the same Li-lon battery.

\section{Acknowledgement}

This work was supported by the PIBITI scholarship and developed in partnership with LALT (Laboratory of Learning in Logistics and Transport) at Unicamp.

${ }^{1}$ Xiong, F. Wireless Temperature Sensor Network based on DS18B20, CC2420, MCU AT89S52. 2015

${ }^{2}$ FREITAS, Tatiana. Mundo desperdiça $30 \%$ dos alimentos produzidos. Disponível em <http://www1.folha.uol.com.br/mercado/2014/07/1488819mundo-desperdica-30-dos-alimentos-produzidos.shtml> Acesso em 03 de jul de 2017. 